\title{
Avaliação Numérica da Exposição à Irradiação Eletromagnética em HF por Operadores de Rádios para Comunicações Táticas
}

\author{
Arthur Henrique Góes Samary e Maurício Henrique Costa Dias ${ }^{1}$
}

\begin{abstract}
Resumo-Rádios HF são um recurso de grande valia para as Forças Armadas de um modo geral, mesmo nos dias de hoje, em que a disponibilidade de dispositivos de comunicações com grande capacidade de transmissão de informação é cada vez maior. Um cenário de uso comum em comunicações táticas é o do operador carregando um transceptor HF nas costas, com potência típica de $10 \mathrm{~W}$. O presente trabalho investiga se esta situação típica representa algum risco à saúde do operador pela exposição à irradiação de RF. $O$ problema do caso analisado é o fato do operador se encontrar na condição de campo próximo da antena, o que invalida o uso de expressões simplistas de avaliação baseadas em condição de campo distante e atenuação no espaço livre. Este artigo utiliza um software baseado no método dos momentos para calcular os campos elétrico e magnético que incidem sobre o operador. A avaliação da exposição é feita comparando os limiares de segurança das principais recomendações sobre o assunto - as diretrizes do ICNIRP e a norma C95.1 do IEEE, com valores calculados.
\end{abstract}

Palavras-Chave-Comunicações táticas, HF, efeitos biológicos, irradiação não ionizante, campo próximo.

Abstract-HF radios are a valuable resource for any armed force, even today, when broadband communications are easily available. A typical scenario in tactical communications comprises mobile manpack operation, transmitting at power levels around $10 \mathrm{~W}$. This work investigates if this $\mathrm{HF}$ tactical operator is actually subject to any kind of biological hazard due to the absorption of the radiated RF signal. In this situation, such assessment may not be carried out based on a simple farfield free space attenuation approach, since the operator is actually in the antenna near field zone. The present work chooses the method of moments to calculate the electric and magnetic near fields and other required parameters, using a simple but adequate enough NEC code software. Such values are compared to the safety exposition levels recommended by the major standards on the subject - the ICNIRP guidelines and the IEEE C95.1 standard.

Index Terms-Tactical communications, HF, biological effects, non-ionizing radiation, near field.

\section{INTRODUÇÃO}

O emprego de rádios HF (High Frequency) ainda é intenso pelas Forças Armadas de um modo geral, seja para

(1) Seç. Eng. Elétrica (SE/3), Instituto Militar de Engenharia (IME), Rio de Janeiro, Brasil,

e-mails: arthurhenrique@uol.com.br,mhcdias@ime.eb.br. comunicações estratégicas ou táticas. Apesar da baixa capacidade de transmissão de informação e das grandes dificuldades impostas pelo canal de propagação naquela faixa, é possível estabelecer enlaces de longa distância com potências de transmissão relativamente baixas, via refração ionosférica [1]. Mesmo considerando-se apenas a componente terrestre de propagação, há alguns cenários táticos em que a faixa de HF apresenta-se como a melhor opção, como por exemplo em ambientes de vegetação densa, como é o caso de muitos cenários típicos no Brasil [2].

Além das baixas taxas de transmissão, outra dificuldade inerente às comunicações em HF é o tamanho das antenas. Lembrando que os comprimentos de onda no espaço livre correspondentes à faixa variam de 10 a $100 \mathrm{~m} \mathrm{(30} \mathrm{MHz} \mathrm{a} 3$ $\mathrm{MHz}$, respectivamente), uma antena ressonante simples como um monopolo de $1 / 4$ de onda precisa ter de 2,5 a $25 \mathrm{~m}$ de comprimento. Praticamente não há alternativa para se operar com mobilidade senão o uso de antenas eletricamente curtas. Como contrapartida indesejável, a eficiência de acoplamento de energia entre o rádio e a antena é baixa [1], [3]-[4].

Em operações táticas terrestres, há duas situações típicas de emprego de rádios HF que normalmente exigem a presença de alguém junto ao aparelho para operar o sistema [1], [5]. O uso de rádios embarcados em viaturas (normalmente leves) é uma delas. Transceptores com potências típicas de até $100 \mathrm{~W}$ são montados em uma plataforma própria dentro da viatura, com a antena posicionada na parte traseira ou por sobre o teto do veículo - a comunicação pode ser feita mesmo em movimento (modo de emprego "móvel veicular").

Para a operação em terra firme, por sua vez, dependendo da situação de emprego, uma das formas mais comuns é o desdobramento de uma estação-rádio fixa no local. Neste caso, antenas eficientes e com alguma diretividade podem ser efetivamente utilizadas, além de amplificadores e casadores de impedância externos, dispositivos auxiliares para transmissão de dados, entre outras facilidades. $\mathrm{O}$ rádio pode ser operado no próprio local, ou através de controle remoto (nomalmente usando cabos).

O outro modo de emprego é o "móvel pessoal", que na verdade pouco se assemelha com o modo homônimo associado ao uso de aparelhos reduzidos que cabem na palma da mão, como celulares e outros transceptores que operam em freqüências mais altas. Dada a necessidade de se operar com antenas curtas, a potência do rádio deve ser alta $(5$ a $20 \mathrm{~W}$ são valores típicos), e o equipamento tem a dimensão similar à de 
uma caixa de sapatos, sem contar a bateria. Estes rádios são denominados comumente de manpack, e usualmente são transportados nas costas do operador.

A proximidade da antena de um rádio de alta potência a seu operador pode gerar questionamentos quanto a potenciais efeitos biológicos da exposição à irradiação de RF (RádioFreqüência). O principal problema que pode advir neste caso é o chamado efeito térmico, que nada mais é que o aquecimento do corpo humano ou de suas partes em função da absorção da energia eletromagnética em níveis muito elevados [6]-[7]. Há várias referências na literatura (leiga e especializada), mais notadamente a partir de meados da década de 90, que discutem, sob vários aspectos, os efeitos da exposição de RF pelo uso de celulares ou pela presença próxima de estações rádio-base[7]. Entretanto, o conhecimento sobre os efeitos da exposição à irradiação de RF já era relativamente grande antes mesmo das discussões em torno dos sistemas celulares; talvez a divulgação daquele conhecimento é que fosse muito restrita até então. Já na década de 60, por exemplo, o IEEE (Institute of Electrical and Electronics Engineers) dispunha de uma norma sobre o assunto - a C95.1 [8]. As discussões oriundas do crescimento significativo do emprego de telefones celulares ajudaram a alavancar as pesquisas sobre o assunto, que passaram a buscar respostas inclusive para questões polêmicas e de difícil averiguação, como os chamados efeitos não-térmicos [6], [9].

Documentos normativos como a C95.1 do IEEE e as diretrizes do ICNIRP (International Commission on NonIonizing Radiation Protection) [10] estabelecem limiares de segurança para a exposição de RF de poucos $\mathrm{kHz}$ até 300 GHz. Para a parte mais alta do espectro, é possível assumir a condição de campo distante da fonte, o que permite análises simplificadas para determinação de distâncias seguras. Algumas normas inclusive já trazem esta formulação para cálculo imediato a partir de parâmetros como freqüência, potência e ganho da antena. No entanto, há situações em que a fonte está muito próxima do ponto que se deseja analisar, como quando se usa um celular junto à cabeça, ou como no caso de interesse deste trabalho, que é a operação de rádios manpack. Nesses casos, é comum o uso de métodos numéricos em eletromagnetismo, tais como o de diferenças finitas no domínio do tempo (FDTD - Finite Difference Time Domain) [11], o de linha de transmissão (TLM Transmission Line Method) [12], entre outros.

Neste contexto, o presente artigo apresenta uma análise teórica da exposição típica à irradiação de RF a que operadores de rádios HF manpack estão sujeitos. Para tal, o método dos momentos (MoM - Method of Moments) [3] é adotado como técnica para se calcular os campos elétricos e magnéticos próximos à antena transmissora em uma situação representativa daquele cenário. Os valores de campo calculados são comparados aos limiares estabelecidos pelo IEEE e pelo ICNIRP para a faixa de HF, a fim de avaliar se, e em que condições, a operação está dentro da faixa de segurança quanto à exposição de RF.

A seção II resume as recomendações pertinentes à faixa de HF encontradas nas principais normas de segurança quanto à exposição a campos eletromagnéticos. A modelagem do problema em questão usando método dos momentos é apresentada na seção III. A avaliação da exposição é tratada na seção IV. Por fim, a seção V conclui o trabalho.

\section{Normas de Segurança para IrRadiações em HF}

$\mathrm{Na}$ literatura atual, os dois documentos normativos mais referenciados sobre limiares de segurança à exposição de RF são a norma C95.1 do IEEE [8] e as diretrizes do ICNIRP [10]. A primeira é adotada nos Estados Unidos, e a segunda em grande parte da Europa e outros países no mundo inteiro. Ambas possuem muitos pontos em comum, mas os limiares recomendados são ligeiramente diferentes. Atualmente, há um esforço em comum para unificação dos limiares de segurança - segundo Repacholi [13], a próxima revisão do ICNIRP deverá contemplar limiares concordantes com os do IEEE, que recentemente (2006) concluiu sua última revisão. Outra iniciativa importante é o projeto EMF (Electromagnetic Fields) da Organização Mundial de Saúde (OMS) [14]. No Brasil, a ANATEL (Agência Nacional de Telecomunicações) é a responsável pela regulamentação que estabelece limites de exposição [15], que basicamente segue as diretrizes do ICNIRP. É prática comum a revisão periódica dessas normas por parte das instituições que as emitem.

De modo geral, tais normas de exposição foram elaboradas para proteger contra os efeitos conhecidos causados pela energia de RF. Até o momento, tais danos estão associados basicamente ao efeito térmico, pois os comitês de especialistas que elaboraram as diretrizes consideram que os relatos indicando potenciais efeitos não-térmicos ainda não constituem evidência científica consistente o suficiente para alterações de maior porte [8], [10], [13]. É importante ressaltar que a irradiação de RF é não-ionizante, e como tal, não possui energia suficiente para ionizar átomos ou moléculas.

Os efeitos térmicos basicamente provocam alteração de temperatura no corpo ou em parte dele. Tais efeitos são observáveis somente sob exposição de alta intensidade. $\mathrm{O}$ calor adicional gerado pela absorção de RF tende a ser dissipado pelo próprio sistema circulatório. $\mathrm{O}$ risco maior ocorre em áreas de baixa vascularização, como as córneas por exemplo [6], [8], [10]. Por sua vez, costuma-se definir os efeitos não-térmicos como aqueles não associados ou não dependentes da geração de calor ou de aumento de temperatura mensurável. Normalmente estão associados à exposição de médio e longo prazos [8], [10], [13].

Os mecanismos de acoplamento dos campos eletromagnéticos de alta freqüência que geram efeito térmico são distintos por sub-bandas de freqüência. Quanto às bandas 
que incluem as freqüências de $\mathrm{HF}(3-30 \mathrm{MHz})$, entre $100 \mathrm{kHz}$ e $20 \mathrm{MHz}$ a absorção de energia se dá principalmente no tronco, pescoço e/ou pernas. De $20 \mathrm{MHz}$ a $300 \mathrm{MHz}$, a absorção se dá em partes do corpo, mas também no corpo inteiro [10].

Tanto ICNIRP quanto IEEE adotam margens expressivas de segurança com relação ao valor de taxa de absorção específica (SAR - Specific Absorption Rate) a partir do qual "distúrbios comportamentais" começam a ser percebidos pelo ser humano. Esta margem é de 10 vezes para a chamada exposição em ambientes controlados, e de 50 vezes em ambientes não-controlados, segundo a C95.1. O ICNIRP adota a mesma postura, embora as denominações sejam outras (exposição ocupacional e exposição do público em geral, respectivamente). Em essência admite-se que pessoas cientes da exposição à irradiação de RF em um determinado ambiente e de seus efeitos irá naturalmente se precaver quanto à exposição próxima ou por tempo excessivo, daí a margem ser menos conservadora que para leigos em geral.

A SAR é o parâmetro dosimétrico original de análise dos efeitos da exposição à RF. Entretanto, a SAR não é de fácil medição ou cálculo teórico. Neste sentido, as normas recomendam como métricas de maior praticidade para avaliação quantitativa da exposição a densidade de potência de onda plana equivalente $(S)$, e as intensidades de campo elétrico $(E)$ e magnético $(H)$. Em especial, para as faixas mais baixas (incluindo $\mathrm{HF}$ ), a condição de campo próximo é bastante comum, e neste caso, a razão $E / H$ deixa de ser constante como em campo distante. Com isso, em campo próximo é necessário avaliar os dois campos.

A Tabela I apresenta os limites de exposição a RF recomendados pelo ICNIRP para exposição ocupacional, na banda entre 1 e $400 \mathrm{MHz}$ (que inclui a faixa de HF). A Tabela II apresenta os limiares análogos definidos pelo IEEE.

TABELA I

Limites do ICNIRP para Exposição OCUPaCional na FaiXa de 1 a $400 \mathrm{MHz}$

\begin{tabular}{|c|c|c|}
\hline Faixa de $\mathbf{R F}$ & $\boldsymbol{E}[\mathbf{V} / \mathbf{m}]$ & $\boldsymbol{H}[\mathbf{A} / \mathbf{m}]$ \\
\hline $1 \mathrm{MHz}$ a $10 \mathrm{MHz}$ & $610 / f$ & $1,6 / f$ \\
\hline $10 \mathrm{MHz}$ a $400 \mathrm{MHz}$ & 61 & 0,16 \\
\hline
\end{tabular}

$f$ em MHz

TABELA II

Limites do IEEE para Exposição OCupacional na Faixa de 1 a $400 \mathrm{MHz}$

\begin{tabular}{|c|c|c|}
\hline Faixa de RF & $\boldsymbol{E}[\mathbf{V} / \mathbf{m}]$ & $\boldsymbol{H}[\mathbf{A} / \mathbf{m}]$ \\
\hline $3 \mathrm{MHz}$ a $30 \mathrm{MHz}$ & $1842 / f$ & $16,3 / f$ \\
\hline
\end{tabular}

$f$ em MHz

O regulamento de exposição da ANATEL [15] traz uma tabela adicional com distâncias mínimas de segurança com relação à antena irradiante. A Tabela III apresenta essas distâncias $(d)$ para as duas faixas já tratadas na Tabela I. Entretanto, tais expressões foram formuladas para a situação de campo distante, usando um fator adicional para contabilizar componentes de reflexão (no solo, por exemplo) que interferem construtivamente com a componente direta [16]. O uso das expressões da Tabela III em condições de campo próximo, como é o caso do problema abordado neste trabalho, não é o mais indicado, portanto.

TABELA III

Expressões para Cálculo de Distâncias Mínimas a Antenas de EstaÇões Transmissoras para Atendimento aos Limites de Exposição Ocupacional da ANATEL

\begin{tabular}{|c|c|}
\hline Faixa de RF & Distância de Segurança [m] \\
\hline $1 \mathrm{MHz}$ a $10 \mathrm{MHz}$ & $d=0,0144 f \sqrt{ } E I R P$ \\
\hline $10 \mathrm{MHz}$ a $400 \mathrm{MHz}$ & $d=0,143 f \sqrt{ } E I R P$ \\
\hline
\end{tabular}

$E I R P$ - Potência Efetiva Irradiada Isotropicamente

\section{Modelagem do Problema Usando Software Baseado em Método dos Momentos}

Uma das implementações mais conhecidas do método dos momentos disponíveis para a comunidade científica em geral são os códigos NEC (Numerical Electromagnetic Codes) [17]. Muitas implementações baseadas em NEC podem ser encontradas na forma de freeware ou comercialmente. Há desde versões mais simples e mais antigas feitas para os sistemas operacionais baseados em MS-DOS e sistemas Unix, a softwares mais sofisticados desenvolvidos e comercializados por companhias como a Pointer Software ou a Nittany para os sistemas operacionais atuais como Windows, Linux ou MacOS

Neste contexto, o software EZNEC foi adotado como ferramenta de apoio para a modelagem do problema. Este software apresenta uma interface de usuário de fácil uso, e dispõe de recursos adicionais de grande utilidade além do usual cálculo de diagrama de radiação. É possível, por exemplo, calcular a resistência de radiação da antena, taxas de onda estacionária, tabelas com valores de campo e, em especial, intensidade de campo próximo (elétrico e magnético). Embora haja versões de códigos NEC que atuem com formas de antenas mais sofisticadas, o padrão é a operação com antenas filamentares, como é o caso do EZNEC. O programa permite, ainda, considerar a presença do solo, condutor perfeito ou não [18].

O seguinte cenário foi idealizado para a avaliação desejada: um operador carregando nas costas um rádio manpack $\mathrm{HF}$, com antena do tipo whip ("chicote") de três metros (3 m) de comprimento, disposta de maneira vertical em relação à terra, a $1,5 \mathrm{~m}$ do chão. Uma potência típica de $10 \mathrm{~W}$ na saída do rádio foi assumida. A Fig. 1, gerada no próprio EZNEC, ilustra o posicionamento relativo da antena. A Fig. 2, por sua vez, apresenta o diagrama de radiação de plano vertical da antena avaliada em $15 \mathrm{MHz}$, considerando uma terra condutora perfeita (na horizontal a antena é onidirecional).

Para que o método dos momentos funcione adequadamente, é conveniente dividir a antena em segmentos 
no mínimo iguais a $1 / 20$ do menor comprimento de onda previsto $(10 \mathrm{~m})$ [3]. Ou seja, cada segmento deveria ter no máximo $0,5 \mathrm{~m}$ de comprimento, e com isso bastariam 6 segmentos para cobrir a antena. Uma folga considerável foi assumida, dividindo a antena em 101 segmentos. Com isso, a análise da irradiação ao redor da antena pôde ser analisada para toda a faixa de HF.

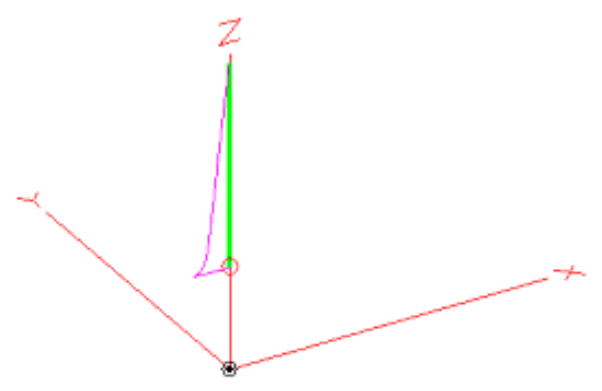

Fig.1. Representação da antena whip assumida.

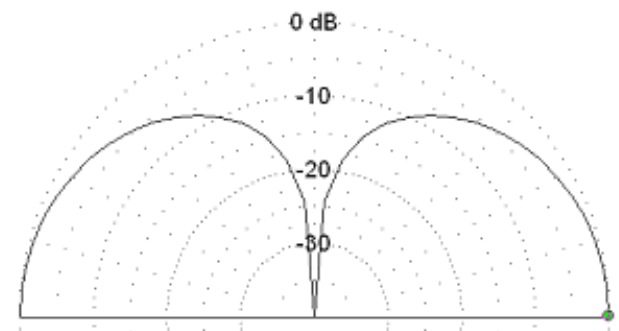

Fig. 2. Diagrama de radiação da antena whip assumida.

Três freqüências foram selecionadas na presente análise, representativas da faixa de $\mathrm{HF}$ como um todo: $3 \mathrm{MHz}, 15$ $\mathrm{MHz}$ e $30 \mathrm{MHz}$. Para cada freqüência as intensidades dos campos elétricos e magnéticos foram calculadas desde uma distância quase infinitesimal a partir da antena (adotou-se 5 $\mathrm{cm}$ ) até a distância de três metros (em passos de $5 \mathrm{~cm}$ ), no plano horizontal, mantendo-se uma mesma altura. Quatro alturas diferentes foram analisadas, a saber: $0,30 \mathrm{~m} ; 1 \mathrm{~m} ; 1,5$ $\mathrm{m}$; e 1,8 m. A idéia foi calcular a intensidade de campo em pontos distribuídos verticalmente, simulando uma pessoa com altura de pouco mais de $1,8 \mathrm{~m}$. De posse da intensidade de campo em quatro pontos na vertical, para cada distância, calculou-se a média, tendo como meta avaliar o acoplamento de corpo inteiro.

Cumpre destacar que a presente modelagem representa uma abordagem simplificada do problema. Apenas a antena e a presença do solo são consideradas. Uma modelagem mais sofisticada e completa deveria incluir também o rádio (ou seu receptáculo) e a pessoa que o opera, pois ambos interagem com as ondas eletromagnéticas emitidas pela antena, dado que se encontram em sua região de campo próximo. Para este tipo de abordagem o método dos momentos deixa de ser uma boa opção (em especial considerando a interação de campos eletromagnéticos com o ser humano). Neste caso, dispondo de capacidade numérica adequada, métodos numéricos que discretizem todo o domínio em estudo são mais apropriados, tais como os métodos: das diferenças finitas no domínio do tempo (FDTD); de linha de transmissão (TLM); e dos elementos finitos (FEM - Finite Element Method) [19].

\section{Avaliação da Exposição à Irradiação de Campo Próximo EM HF}

Como citado anteriormente, as normas C95.1 [8] e as diretrizes da ICNIRP [10] são as duas referências mais respeitadas no que diz respeito a limites de segurança à exposição eletromagnética de RF. Desta forma todos os dados gerados por este trabalho foram comparados com os limiares dessas referências.

As Figs. 3 e 4 apresentam as intensidades médias de campo elétrico calculadas, em comparação com os limiares de segurança para exposição ocupacional (ou em ambiente controlado) do ICNIRP e do IEEE, respectivamente. Os limiares de referência são indicados pelas linhas horizontais tracejadas. Da Tabela I observa-se que os limiares para 15 e $30 \mathrm{MHz}$ são os mesmos, em concordância com a Fig. 3. Percebe-se que as distâncias de segurança segundo o ICNIRP são mais pessimistas que as do IEEE, em especial para as freqüências mais baixas. A Fig. 5 apresenta o cáculo das intensidades médias de campo magnético, comparadas aos limiares do ICNIRP. Percebe-se claramente que as distâncias são menores que as obtidas a partir do cálculo de campo elétrico, o que já era esperado, pois a antena whip tem um comportamento similar ao de um grande "capacitor" para pontos próximos à antena, e com isso, o comportamento é predominantemente elétrico.

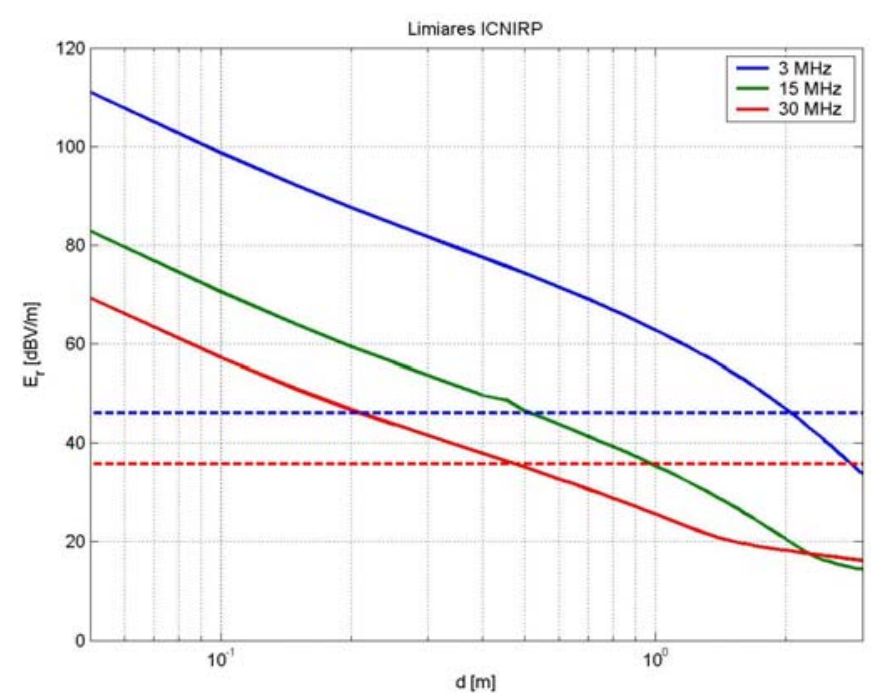

Fig. 3. Intensidade média de campo elétrico $\times$ limiares do ICNIRP. Distâncias de segurança dadas por 2,1 e 0,5 m para 3, 15 e $30 \mathrm{MHz}$, respectivamente. 


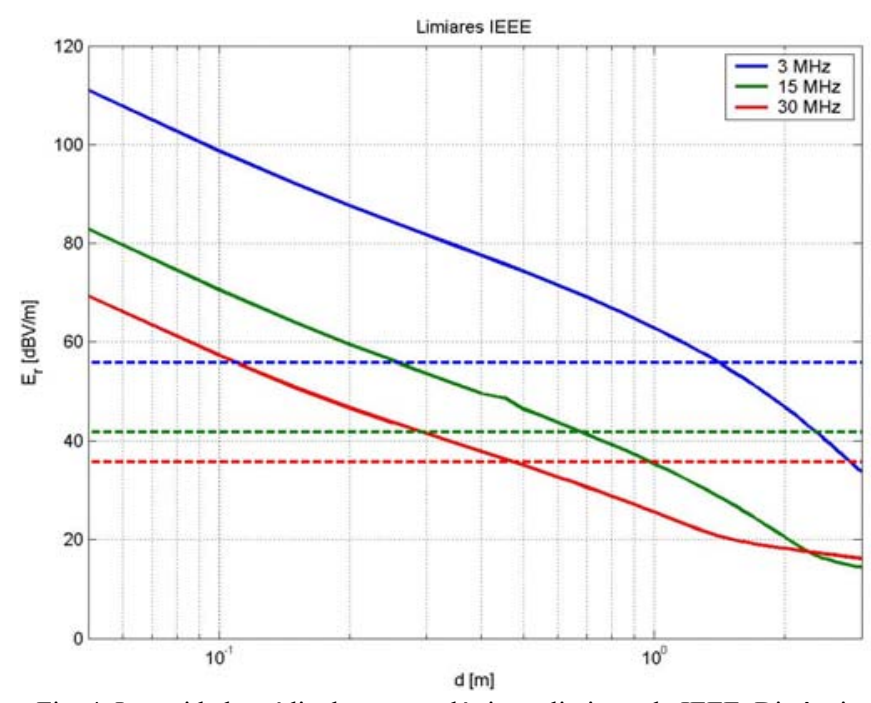

Fig. 4. Intensidade média de campo elétrico $\times$ limiares do IEEE. Distâncias de segurança dadas por $1,2,0,7$ e $0,45 \mathrm{~m}$ para 3,15 e $30 \mathrm{MHz}$, respectivamente.

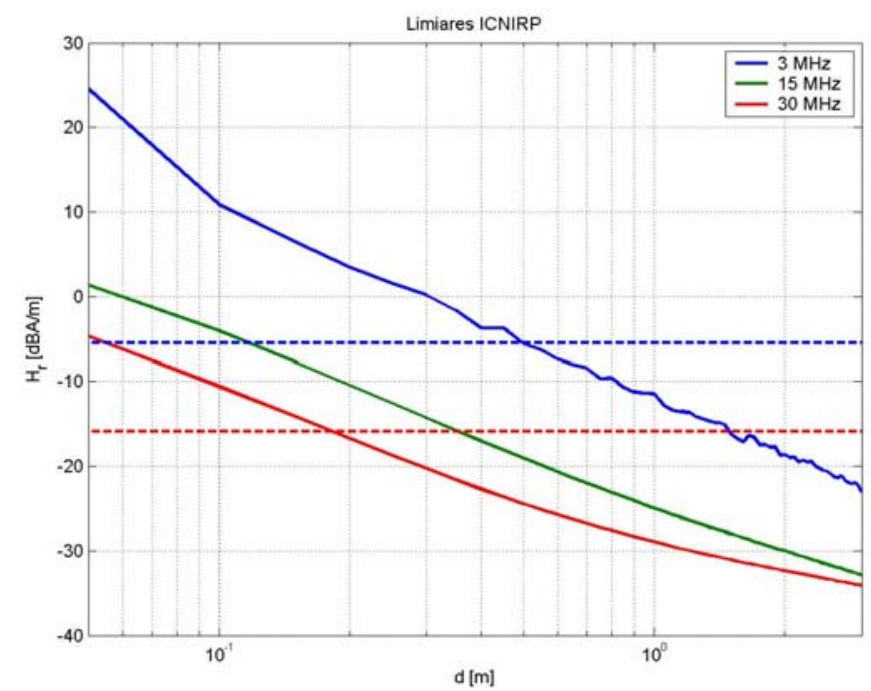

Fig. 5. Intensidade média de campo magnético $\times$ limiares do ICNIRP. Distâncias de segurança dadas por $0,5,0,32$ e $0,17 \mathrm{~m}$ para 3,15 e $30 \mathrm{MHz}$, respectivamente.

Neste ponto da avaliação cabem algumas ponderações relevantes quanto à interpretação literal dos valores apresentados. $\mathrm{Na}$ prática, as Figs. 3 a 5 apresentam estimativas superestimadas das distâncias de segurança. Há dois argumentos fundamentais que justificam tal afirmação: a eficiência do casamento ou acoplamento de impedância entre a saída do rádio e a antena; e a eficiência resistiva da antena. Como previamente mencionado, o operador de rádio manpack precisa usar antenas eletricamente curtas. A resistência de radiação dessas antenas é baixa, podendo ser comparável ou até menor que a própria resistência de perdas da antena [3]-[4]. Neste caso, portanto, a eficiência resistiva da antena é baixa, e com isso o campo efetivamente irradiado é menor que o calculado. Adicionalmente, é tecnologicamente complicado realizar casamento de impedância com valores baixos de resistências, como é o caso. Alguns fabricantes de acopladores informam que a eficiência de casamento para antenas mais curtas em freqüências menores pode ser tão baixa quanto $15 \%$ [20].

A fim de quantificar ao menos um dos parâmetros redutores do acoplamento de energia entre o rádio e a antena, estimou-se a eficiência resistiva da antena para a análise em questão, assumindo um valor típico para a resistência de perdas da antena $R_{l}=2 \Omega$ [4]. As resistências de radiação calculadas pelo EZNEC foram $0,17,4,5$ e $24,98 \Omega$ em 3,15 e $30 \mathrm{MHz}$, respectivamente. Com isso, as eficiências nas três freqüências foram 0,08, 0,69 e 0,93, respectivamente. A Fig. 6 apresenta os valores de campo elétrico corrigidos pelas eficiências respectivas em comparação com os limiares do ICNIRP. A redução da distância de segurança para $3 \mathrm{MHz}$ fica bastante clara, e representa um valor mais realista que o da Fig. 3, sem contar a potencial perda no casamento de impedâncias.

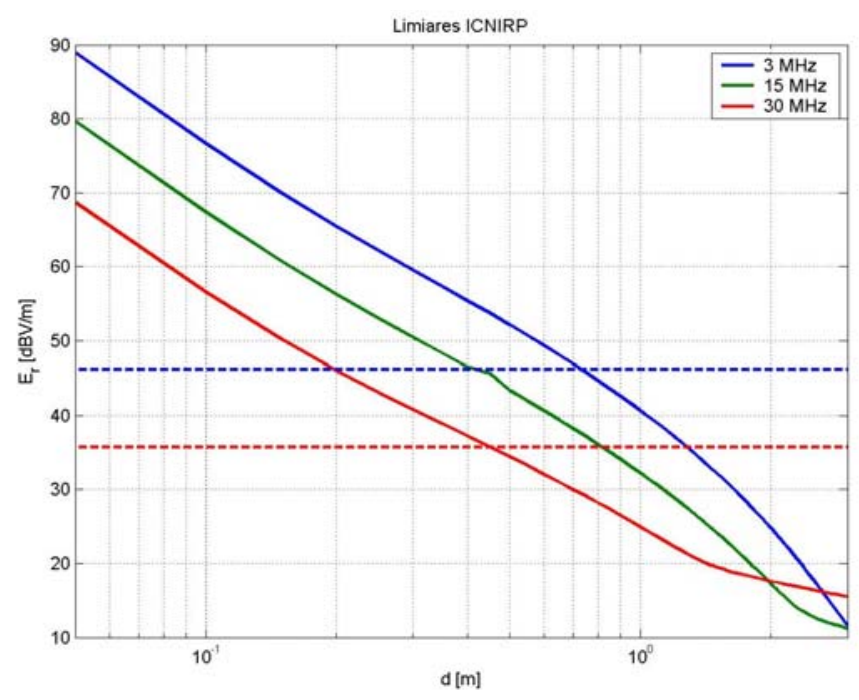

Fig. 6. Intensidade média de campo elétrico $\times$ limiares do ICNIRP, considerando eficiência resistiva. Distâncias de segurança dadas por 0,71, 0,8 e $0,42 \mathrm{~m}$ para 3,15 e $30 \mathrm{MHz}$, respectivamente.

A título de comparação, foram calculadas também as distâncias de segurança segundo a metodologia da Tabela III. Para $3 \mathrm{MHz}, d=0,24 \mathrm{~m}$, e para as duas outras freqüências $d$ $=0,78 \mathrm{~m}$. Ou seja, apesar de partir de uma premissa equivocada (hipótese de campo distante), a ordem de grandeza dos valores estimados foi compatível com a dos valores calculados de maneira mais formal.

As distâncias de segurança obtidas da Fig. 6 são na realidade um pouco menores em função dos argumentos já citados. Entretanto, não são completamente desprezíveis, pois têm a ordem de grandeza das dimensões horizontais do corpo 
humano. É possível, portanto, que na prática operadores transmitindo na potência máxima durante longos períodos possam sentir algum desconforto adicional em função do efeito térmico previsto.

\section{Conclusão}

Este trabalho apresentou uma avaliação da exposição à irradiação de RF por operadores de rádios $\mathrm{HF}$ do tipo manpack. Para tal, adotou-se o método dos momentos como ferramenta para calcular campos elétricos e magnéticos próximos à antena, onde as aproximações usuais de campo distante não valem. Usando um software baseado naquele método, foi possível computar valores de campos para uma situação típica representativa.

Os valores obtidos a partir da ferramenta disponível foram comparados aos limiares de segurança do IEEE e do ICNIRP. Para a situação analisada, em que uma antena whip curta foi utilizada, o campo elétrico foi o determinante da condição de segurança. Valores mais realistas foram também apresentados, considerando uma estimativa da eficiência de acoplamento resistivo na antena. Ainda assim, verificou-se que operadores nesta situação podem estar sujeitos a exposições pouco acima dos limiares recomendados.

A análise apresentada não é a mais realista ou acurada, como já argumentado ao fim da seção III, mas é mais confiável que a análise elementar que assume campo distante e perda no espaço livre. Uma abordagem ainda mais direta é calcular a SAR no corpo do operador, por simulação. Obviamente, a complexidade desta modelagem é ainda maior que o cálculo dos campos tão somente. Em última instância, a realização de medidas de campo ao redor de um transmissor em funcionamento é outra abordagem recomendável.

\section{Agradecimentos}

O primeiro autor expressa sua gratidão à Fundação Ricardo Franco e ao Banco Santander pelo apoio financeiro.

\section{REFERÊNCIAS}

[1] E. Johnson, R. Desourdis, G. Earle, S. Cook and J. Ostergard, Advanced High-Frequency Radio Communications, Artech House, 1997.
[2] J. C. R. Dal Bello e M. S. Assis, "Comunicações Táticas na Região Amazônica - $1^{a}$ Parte", Revista Militar de Ciência e Tecnologia, vol. IX, no. 3, pp. 12-20, 1992.

[3] C. A. Balanis, Antenna Theory: Analysis and Design, $2^{\mathrm{a}}$ ed, Wiley, 1997.

[4] J. D. Kraus, Antennas, $2^{\text {nd }}$ Ed, McGraw-Hill, 1988.

[5] M. R. Frate e M. Ryan, Electronic Warfare for the Digitized Battlefield, Artech House, 2001.

[6] A. A. A. de Salles, "Biological effects of microwave and RF", in: 1999 SBMO/IEEE MTT-S International Microwave and Optoelectronics Conference, Rio de Janeiro - RJ, vol. 1, 1999, pp. 51-56.

[7] M. H. C. Dias e G. L. Siqueira, "Considerações sobre os Efeitos à Saúde Humana da Irradiação Emitida por Antenas de Estações RádioBase de Sistemas Celulares", Telecomunicações, vol. 5, no. 1, pp. 4154, Jun. 2002.

[8] IEEE C95.1-2005, Standard for safety levels with respect to human exposure to radio frequency electromagnetic fields, $3 \mathrm{kHz}$ to $300 \mathrm{GHz}$, 19 Abr 2006

[9] E. Litvak, K. R. Foster, e M. H. Repacholi, "Health and Safety Implications of Exposure to Electromagnetic Fields in the Frequency Range $300 \mathrm{~Hz}$ to $10 \mathrm{MHz}$ ", Bioelectromagnetics, vol. 23, pp. 68-82, 2002.

[10] ICNIRP, "Guidelines for limiting exposure to time-varying electric, magnetic, and electromagnetic fields (up to $300 \mathrm{GHz}$ )," Health Physics, vol. 74, no. 4, pp. 494-522, Abr. 1998.

[11] A. A. de Salles, C. R. Fernández e M. Bonadiman, "Simulações da SAR na cabeça e antenas planares para telefones móveis", Revista Brasileira de Engenharia Biomédica, vol. 19, no. 2, pp. 77-90, Ago. 2003

[12] H. A. D. Almaguer e A. Raizer, "Aplicação do método TLM no estudo da interação dos campos irradiados por telefones celulares com a cabeça humana", Revista Brasileira de Engenharia Biomédica, vol. 18, no. 1, pp. 17-25, Jan/Abr 2002.

[13] M. H. Repacholi, Campos Eletromagnéticos de Baixa Freqüência e a Saúde: Estado da Arte e Recomendações da Organização Mundial de Saúde, apresentação no Auditório do CEPEL, Rio de Janeiro - RJ, 27 Set 2005 .

[14] Sítio The International EMF Project - World Health Organization, em http://www.who.int/peh-emf/project/en/

[15] ANATEL, Resolução 303/2002 - Regulamento sobre limitação da exposição a campos elétricos, magnéticos e eletromagnéticos na faixa de radiofrequiências entre $9 \mathrm{kHz}$ e $300 \mathrm{GHz}$, em www.anatel.gov.br.

[16] R. F. Cleveland Jr., D. M. Sylvar e J. L. Ulcek, Evaluating Compliance with FCC Guidelines for Human Exposure to Radiofrequency Electromagnetic Fields, FCC-OET Bulletin 65, Edition 97-01, Ago. 1997

[17] D. Adler, "Information on the history and availability of NEC-MoM codes for PC's \& UNIX," Applied Computational Electromagnetics Society Newsletter, vol. 8, no. 3, pp. 8-10, Nov. 1993.

[18] Sítio do EZNEC,em www.eznec.com.

[19] M. N. O. Sadiku, Numerical Techniques in Electromagnetism, $2^{\text {nd }} \mathrm{Ed}$, CRC Press, 2001.

[20] Especificação técnica do acoplador de antena RF-382, em www.harris.com. 